\title{
Hyperscanning: a valid method to study neural inter brain underpinnings of social interaction
}

\author{
Artur Czeszumski ${ }^{1, *}$, Sara Eustergerling ${ }^{1}$, Anne Lang ${ }^{1}$, David Menrath ${ }^{1}$, Michael Gerstenberger ${ }^{1}$, \\ Susanne Schuberth ${ }^{1}$, Felix Schreiber ${ }^{1}$ Zadkiel Zuluaga Rendon $^{1}$, and Peter König ${ }^{1,2}$ \\ 1 Institute of Cognitive Science, Universität Osnabrück, Osnabrück, Germany \\ 2 Institut für Neurophysiologie und Pathophysiologie, Universitätsklinikum Hamburg-Eppendorf, \\ Hamburg, Germany \\ * aczeszumski@uni-osnabrueck.de
}

\begin{abstract}
Social interactions are a crucial part of human life. Understanding the neural underpinnings of social interactions is a challenging task that the hyperscanning method is trying to tackle in the last two decades. Here, we review the existing literature and evaluate the current state of the hyperscanning method. We review the type of methods (fMRI, M/EEG, fNIRS) that are used to measure brain activity from more than one participant simultaneously and their pros and cons for hyperscanning. Further, we discuss different types of analyses that are used to estimate between brain networks and synchronization. Lastly, we present results of hypercanning studies in the context of different cognitive functions and their relations to social interactions. All in all, we aim to comprehensively present methods, analyses, and results of the last twenty years of hyperscanning research.
\end{abstract}

\section{Keywords:}

hyperscanning, social cognition, joint action, EEG, MEG, fMRI, fNIRS, social interactions

\section{Introduction}

The importance of social interactions for the development and maintenance of the human self was already highlighted in Greek philosophy and has been discussed ever since. Nevertheless, the field of cognitive neuroscience has only started to investigate brain activities during social interactions in the last decades. Typically, only the brain of one of the involved participants and, therefore, only one part of dyadic or group interaction was recorded at a time. Thus, the insights such experiments may 
provide are limited. To examine social interactions as a whole, the idea of hyperscanning, i.e., measuring the activity of multiple brains simultaneously, has originated. The significant advantage of this technique is that it allows the investigation of real-time dynamics between two or more interacting brains (Hari et al. 2013 ; Hari and Kujala, 2009). In contrast to classic experimental paradigms that measure the brain activity of single participants during social interaction, simultaneously measuring the brain activity of several interacting participants allows for the investigation of intra- and inter-brain neural relations (Schilbach et al., 2013). Hyperscanning techniques thus offer a new approach to account for the complexity of joint action, i.e., its spontaneity, reciprocity, and multimodality, which constitutes a big challenge for its neuroscientific examination.

In the current paper, we review existing literature and evaluate the current state of the hyperscanning method. We perform extensive literature research to identify the most critical peer-reviewed studies that used hyperscanning as a method to investigate human social cognition. In our review, we have two primary goals. First, we review the methods and types of analysis that are used in the hyperscanning field. Second, we review cognitive functions and their neural underpinnings that are investigated with the hyperscanning method.

\section{Type of methods}

In the last century, a large variety of methods to measure brain activity have been developed. The most popular ways to measure brain activity used in the cognitive neuroscience field are Electroencephalography (EEG) (Luck and Hillyard, 1994), Magnetoencephalography (MEG) (Baillet, 2017), Functional magnetic resonance imaging (fMRI) (Eisenberger, 2003), and functional near-infrared spectroscopy (fNIRS) (Ferrari and Quaresima, 2012). Each of them has its advantages and disadvantages that help us to understand different brain functions. Primarily when focused on Hyperscanning, their specific assets, like temporal and spatial resolution, or mobility, are of value. We review here all of these in the context of Hyperscanning research.

\section{fMRI}

Functional magnetic resonance imaging (fMRI) is a method that indirectly measures neural brain activity. Namely, it measures it by detecting changes associated with blood flow, which is the blood-oxygen-level-dependent (BOLD) contrast (Glover, 2011). Since the last decade of the 20th century, fMRI became one of the most popular methods used in cognitive neuroscience. Its most important advantage is the spatial resolution. Standard fMRI scanners estimate brain activity with a spatial resolution of $3 \mathrm{~mm}$, specialized instruments push the limits towards the sub-millimeter range.

In comparison to other methods discussed below, it is the best method to answer questions where in the brain, something happened. Additionally, it is the non-invasive method of choice for measuring deep brain structures. However, because it uses blood flow to estimate neural activity, its 
temporal resolution does not compare to M/EEG (Glover, 2011). Moreover, to measure the BOLD signal, participants are required to stay stable in laying position within a scanner (Figure 1 (A)). This low mobility of experimental tools makes it not suitable for investigating social interactions in naturalistic and ecologically valid setups. Despite low mobility, the first-ever hyperscanning study was an fMRI study. Montague (2002) performed a successful feasibility study to link participants in two scanners. To tackle the problem that two scanners are required to complete a study: King-Casas (2005) conducted a study using scanners in Texas and California linked via Internet. Afterward, other studies were performed in facilities that posses two scanners; however, until now, only a few studies tried to investigate social interaction with the fMRI hyperscanning method (Abe et al., 2019 Koike et al., 2019, 2016; Saito et al., 2010; Schippers et al., 2010; Spiegelhalder et al., 2014, Tanabe et al. 2012, Tomlin, 2006; Tomlin et al., 2013). One reason for that might be difficulty in creating experimental paradigms that involve interaction between participants without movement and communication. Another reason might be that the complexity of fMRI data requires the development of new types of analysis that are suitable to answer questions about between brain relations. It is sensible to say that the value of each of these studies is excellent, and more studies are required because fMRI has a good spatial resolution. Furthermore, this method could be of great value if combined with EEG to surmount poor temporal resolution (Koike et al., 2015).

\section{EEG/MEG}

One of the oldest methods to measure activity in the brain is electroencephalography (EEG). In comparison to fMRI and fNIRS, it measures neural activity directly by recording electrical activity with the use of electrodes placed on the scalp (Figure 1 (B)). Therefore, it is not dependent on blood oxygenation, and its temporal resolution is higher than other methods (Michel and Brunet, 2019). However, because electrodes are placed on the scalp, it is best suited for investigating the cerebral cortex and not deep brain structures. Classically, EEG was considered a low mobility tool because it required strict control of movements and surroundings of participants, which limits it to the lab environment. However, in the last years, the development of new technologies allowed for improvement in mobility by creating mobile EEG systems (Melnik et al., 2017). Such systems are a great tool to study social interactions. Even though fMRI was the first method used to perform a hyperscanning study, presently, it is EEG that is the most common method used to conduct hyperscanning experiments. Its popularity comes from its most important advantage, temporal resolution. Studies of social interaction that unfold on a fast scale require a method that is sensitive to it. Until now, only EEG can account for changes in neural processing on a millisecond scale while two or more humans perform an interactive task together. The high temporal resolution allows for a more precise and different type of between brain analysis. Another advantage of EEG for hyperscanning studies is that it is easier to measure more than just two heads at the same time, as demonstrated by Dikker (Dikker et al., 2017). The relatively low price of EEG systems and the availability of mobile systems are key advantages. Early EEG hyperscanning research was conducted in the lab with full control of the environment and traditional paradigms (Babiloni et al., 2007alb). 
However, with further developments, more interactive and naturalistic paradigms, like playing guitars (Lindenberger et al., 2009) or romantic kissing (Müller and Lindenberger, 2014), were proven to be feasible. In recent years, another technology, that can be combined with EEG, was developed and implemented to use in research. Namely, virtual reality (VR) (Cipresso et al., 2018, Ehinger et al. 2014 Oliveira et al. 2016) is becoming more and more present in the scientific community. It allows for creating naturalistic paradigms that are fully controlled by the experimenter. This, in combination with the EEG, might be a great tool to study social interactions.

It is worth it to mention that, magnetoencephalography (MEG), a method with similar characteristic to EEG but lower mobility was also proven to be feasible for hyperscanning measurements (Baess et al. 2012) and so far used in a study that combined it with EEG to study verbal interactions (Ahn et al. 2018). Moreover, this method was also utilized to study the interaction between mothers and children (Hirata et al., 2014, Levy et al., 2017). Recently, Boto et al. (2018) developed a mobile MEG system. Therefore, we can expect more MEG hyperscanning studies in upcoming years.

\section{fNIRS}

The last neuroimaging method that we discuss is functional near-infrared spectroscopy (fNIRS). Similarly to fMRI, it measures brain activity indirectly and uses the contrast between oxygenated and de-oxygenated hemoglobin, and similarly to EEG, it can best measure superficial brain areas with a low spatial resolution $(1 \mathrm{~cm}$ ) (Scholkmann et al., 2013) (Figure 1 (C)). Moreover, its temporal resolution is lower than that of EEG and varies between 0.1 to 1 second Quaresima and Ferrari 2019). Despite these limitations, fNIRS is widely used in cognitive neuroscience for its mobility and resistance to motion artifacts. In comparison to other methods discussed here, the signal measured with fNIRS is not strongly influenced by the movement of participants. This feature allows for creating experimental paradigms that resemble real-life situations more closely than classic studies. In the case of studying social interaction that involves actions from participants, it is a critical feature that is required. The first hyperscanning fNIRS study was conducted by Funane et al. (2011) and used a simple tapping synchronization task to investigate the coherence of neural activity between two brains. Since then, many researchers adopted hyperscanning fNIRS in various types of paradigms to study social interactions (Scholkmann et al., 2013). One particularly interesting study was conducted by Nozawa et al. (2016). It involved groups of participants (four) tested in a naturalistic setting (cooperative communication). Furthermore, a recently developed fNIRS system for babies allows for investigating brain functions related to parent-child interaction Reindl et al. 2018). Such experiments are proof of the concept that studying neural between brain underpinnings is feasible, and it brings new insides into understanding human cognition. 


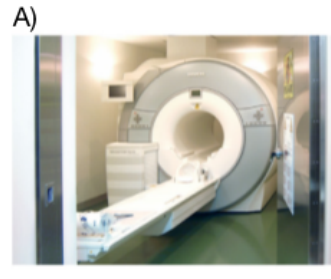

B)

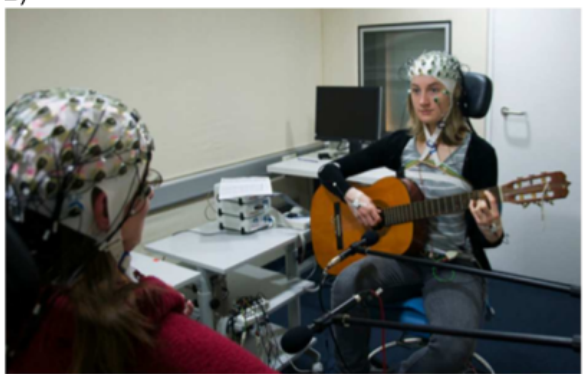

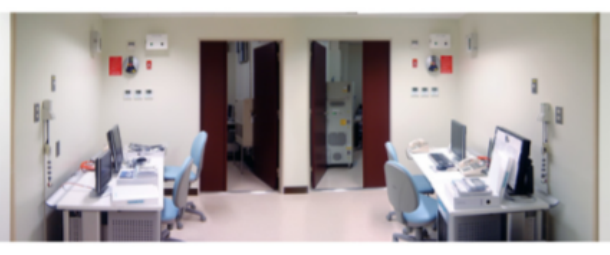

C)

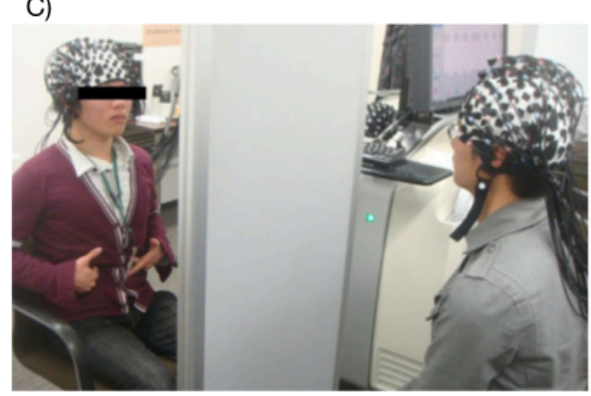

Figure 1. Neuroimaging methods used in Hyperscanning. (A) From Koike et al. (2019). View of the dual fMRI facility used to study mutual gaze. (B) From Sanger et al. (2012). EEG measurement of two guitar players. (C) From Osaka et al. (2015). fNIRS set up used to study cooperative singing.

\section{Type of analysis}

Analysis and interpretation of hyperscanning data is a challenging task. First, an intra-brain type of analysis has to be adjusted to inter-brain data, or even new types of analysis have to be developed. Second, it is challenging to separate inter-brain relations related to identical stimuli presented to both participants from relations that represent between brain networks. This latter issue is often addressed by comparing real participant pairs with randomly selected pairs and permutation analysis (e.g., Bilek et al. (2015)). The types of analyses applied to hyperscanning data can be separated into different categories. There are various coupling measures, correlation and dependence analyses, graph theory measures, and analysis of information flow. In this section, we discuss all these types of analyses in sequence.

\section{Coupling/connectivity measures}

The most common methods to estimate the strength of coupling/connectivity between brains have been previously used to study single brains. They are based on second-order measures calculated in the Fourier domain. They differ in the technical details of combining different frequencies and the kind of normalization. That is, like phase-locking value (PLV), phase lag index (PLI), or phase coherence have been adopted to estimate between brain couplings. PLV measures how two signals (in case of hyperscanning coming from two different brains) are phase-locked in the observed time window. PLV is equal to 1 when phases are perfectly synchronized in a specific 
frequency and to 0 when they are unsynchronized. This measure was used in multiple EEG hyperscanning studies. They investigated cortical synchronization while two participants tried to imitate their hand (Dumas et al., 2011, 2010) or finger movements (Yun et al., 2012)(Figure 2 (A)) during a coordinated time estimation task (Mu et al. 2016), during speaking and listening (Pérez et al., 2017), and cooperative decision-making task (Hu et al. 2018). Another similar measure, also related to phase synchronization, PLI, was used in studies investigating coordinated behavior in guitar players (Lindenberger et al., 2009: Sänger et al., 2012) and also in verbal interaction task with the use of both EEG and MEG (Ahn et al., 2018). Phase coherence is another method of estimating cortical synchronization within or between brains that are related to the phase of neural oscillations. It is a measure of similarity between two signals, and there is more than one way of quantifying it. Different variations of phase coherence were used in hyperscanning experiments. Notably, in studies mentioned above, that investigated guitar players (Lindenberger et al. 2009: Müller et al., 2013. Sänger et al. 2012, 2013), and also romantic partners while kissing (Müller and Lindenberger, 2014). Moreover, the latter study estimated also cross-frequency couplings between brains.

Wavelet transform coherence is a related method to measure the coherence of two signals. It was developed to analyze the geophysical time series (Grinsted et al., 2004). However, it finds its application as well in neuroscience, especially in analyzing fNIRS hyperscanning studies. Since one of the first fNIRS studies (Cui et al. 2012), it was used in the following experiments. As it is the most common method that is used to analyze inter-brain synchrony with fNIRS, it is as well the most common analysis method within all hyperscanning studies (Cheng et al., 2015, Cui et al., 2012 Dommer et al., 2012, Hirsch et al., 2017; Holper et al., 2012, Hu et al., 2017, Ikeda et al., 2017, Jiang et al., 2015, 2012; Liu et al., 2016b; Nozawa et al., 2016, Osaka et al., 2014, 2015, Pan et al., 2017, 2018, Tang et al., 2016, Wang et al., 2019, Zhang et al., 2017).

All coupling/connectivity measures mentioned in this section are measures of similarity between two neural signals coming from different brains. This similarity is interpreted as synchrony between brains in these studies.

\section{Correlation and dependence analysis}

Another way of measuring synchrony between brains is by estimating the correlation between signals coming from two brains. Within the hyperscanning literature, we found different types of correlation measures applied to EEG, fNIRS, and fMRI data. Due to the low temporal resolution of fMRI, coupling measures discussed in the previous chapter could be used only in the very-low-frequency range, typically not associated with information processing in the brain. Therefore, the relation between two measured brains is often estimated with the use of linear dependence. It is not the BOLD signal itself that is used for correlation analysis, but regression model coefficients are representing activations in different tasks. These types of analyses were applied in research investigating mutual gaze, shared attention, and cooperation in the joint force production task (Abe et al., 2019; Koike et al., 2019, 2016, Saito et al., 2010)(Figure 2 (B)). Correlations found in these studies were interpreted as neural synchronization between brains. 
Further, two studies focused on verbal communication between participants and used correlation of BOLD activity to predict the flow of information between the sender and perceiver Anders et al. 2011) and synchronization of brain activity between interlocutors (Spiegelhalder et al., 2014). To further extend dependence analysis, cross-correlation in combination with ICA decomposition of the BOLD signal was used in studies focused on joint attention in participants with borderline personality disorder and healthy participants (Bilek et al. 2015,2017$)$. There, the cross-correlation between two brain signals was interpreted as information flow.

Correlation measures are also applied to EEG hyperscanning data. Namely, we identify studies using correlation as a measure of between brain synchronization in different paradigms. Moreover, different aspects of EEG signals were used for correlation analysis. Correlation between different frequencies (theta and alpha) was used to investigate the coordination of speech rhythm (Kawasaki et al. 2013) as well as differences between interactions between strangers and couples in alpha, beta, and gamma (Kinreich et al., 2017). Furthermore, the total independence analysis (Wen et al., 2012) was used in a study that investigated between brain synchronization in a class environment on a group of students (Bevilacqua et al., 2019, Dikker et al., 2017). This analysis was used to predict classroom dynamics and engagement.

Lastly, two fNIRS experiments applied correlation analysis to estimate synchrony between brains in tasks that require cooperation or competition between participants (Funane et al. 2011, Liu et al. 2015).

\section{Graph Theory measures}

Between brain networks can also be quantified with graph theory measures. Up to today, there are only studies that used graph theory measures on EEG hyperscanning data. Graph theory measures focus on different aspects of between brain networks. Within existing hyperscanning literature, we found studies that focused on links between brains and modularity of networks while participants performed the decision making task (De Vico Fallani et al. 2010). Moreover, different graph theory measures were used to investigate between brain networks in guitar players.

Small-worldness of between brain networks was enhanced during musical coordination (Sänger et al. 2012 ), the topology of between brain networks was dependent on frequency and was more regular at higher frequencies (Müller et al. 2013) (Figure 2 (C)). Additionally, the directionality of between brain networks was used to predict leaders and followers in guitar players (Sänger et al., 2013). In another study, the dimensionality of between brain networks was investigated in combat cooperation tasks (Dodel et al., 2011). All mentioned measures suggest that neural synchrony can be estimated with graph theory measures and that these measures extend our understanding of between brain networks.

\section{Information flow}

Apart from synchrony, similarity, or network properties, one can focus hyperscanning analysis on 
the flow of information from one brain to another. Such studies require estimating causal links between brains. Methods that are used to determine such causal links are Granger Causality and its equivalent in a frequency domain Partial Directed Coherence (PDC). In the EEG hyperscanning literature, these methods were applied to estimate links between brains of cooperating pilots (Astolfi et al., 2012, 2011), and results suggested that causal links are stronger during increased cooperative behavior. Similarly, increased causal links between the brains of participants were found in cooperative and altruistic behaviors in decision-making tasks (Ciaramidaro et al., 2018,

De Vico Fallani et al. 2010). Furthermore, one fMRI and one fNIRS study focused on causal links between brains. Schippers et al. (2010) studied such links in gesture communication with the use of fMRI, and Pan et al. (2017) used fNIRS to explore causal relationships between brains of cooperating lovers. The casual links between brains can be estimated with methods that we discussed here; however, important question what are the neural substrates that allow for information flow between brains has to be answered.

Taken together, in this section, we reviewed different methods and types of analysis that are used in the hyperscanning field. A variety of techniques and analysis suggests that hyperscanning is a new and valuable part of the cognitive neuroscience field. However, in many cases, the advantages and disadvantages of a specific method are not that obvious. Further, at least in part, we consider the growing variety of techniques used as exploratory, and it has to be investigated whether they relate to the same set of physiological processes.

\section{Cognitive functions}

\section{Coordination and Synchronization}

The investigation of interpersonal coordination of actions that includes mutual entrainment or synchronization is one of the fields most predestined for hyperscanning. Simultaneously measuring the brain activity of interacting subjects allows real-time access to the reciprocal coupling of neural processes that enable interpersonal movement synchronization within a millisecond time scale.

Experimental paradigms are addressing the connection between interpersonal neural dynamics and behavioral synchronization span from minimalistic button-pressing tasks to complex naturalistic settings like joint music playing. In minimalistic tasks, different parameters such as visual contact, feedback, and mode of synchronization (in-phase vs. antiphase) can be manipulated easily. Additionally, several studies compare the degree of behavioral synchronization between human-human and human-computer (metronome) couples (Hu et al., 2017, Konvalinka et al., 2014, Mu et al., 2016), in order to extract the social aspect of the interaction. Such setups enable the examination of various aspects of action coordination and synchronization while controlling the effects of a shared sensory environment.

One conventional paradigm used to study coordinated behavior, and it is neural underpinnings, requires participants to perform only one temporally synchronized button press after a self- or 

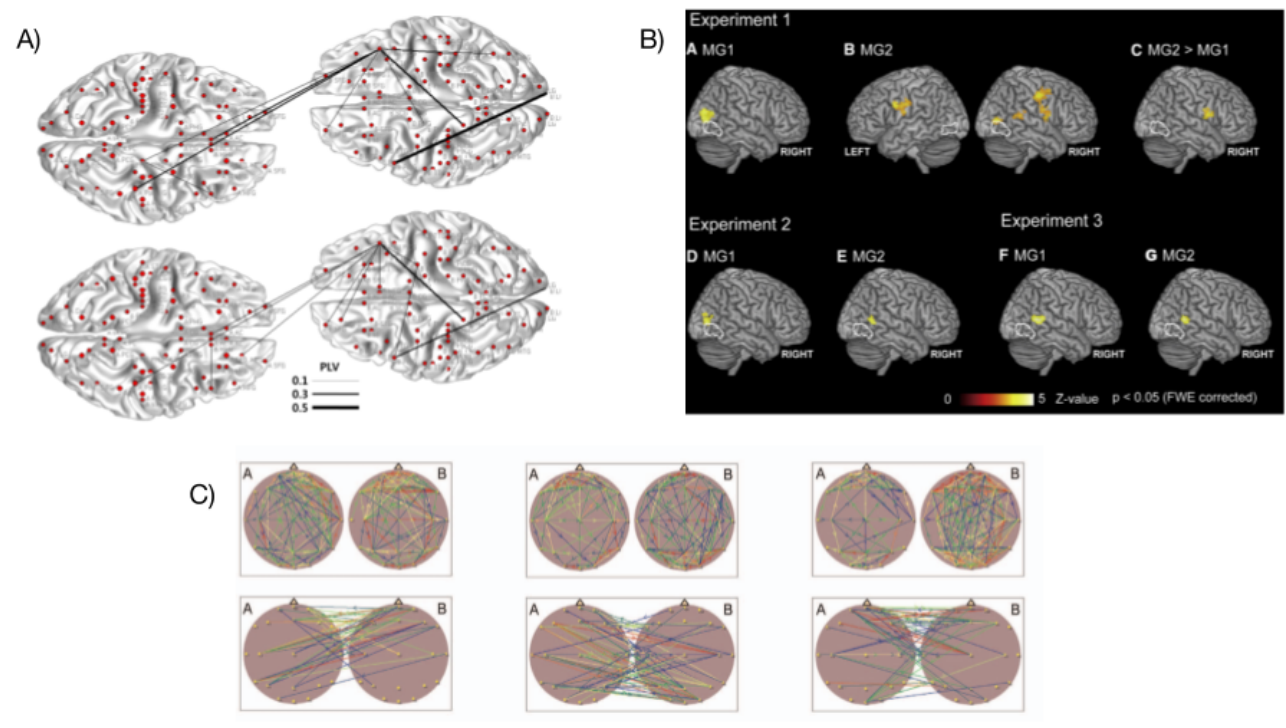

Figure 2. Analysis methods used in hyperscanning to investigate between brain relationships. (A) From Yun et al. (2012). Phase synchrony was used as a coupling measure to investigate between brain connections in implicit coordination task. Topography of the phase synchrony (PLV) between different regions of interest of two participants are presented for theta $(4-7.5 \mathrm{~Hz})$ and beta $(12-30 \mathrm{~Hz})$ oscillations. (B) From Koike et al. (2016). Between brain synchronization estimated with correlation. (C) From Muller et al. (2013). Brain topography maps illustrating significant connection within and between the brains. Example of graph theory measures applied to analyze synchronization during musical improvisation on the guitar.

predefined time interval has passed. As a result of this, better performance was related to higher inter-brain coherence in frontal areas as well as to stronger social connectedness in the dyad (Funane et al., 2011; Hu et al., 2017, Mu et al., 2016, Pan et al., 2017).

Another paradigm used continuous tapping or finger/hand movement, allowing additional insights into the time course and the dynamics of synchronization. Tognoli et al. (2007) found that the spontaneous transition from uncoordinated to coordinated rhythmic movements under vision went along with specific EEG rhythms in the alpha mu range at right centro-parietal sides. Dumas et al. (2010) took a between-brain-approach, using the Phase Locking Value (PLV) across a variety of different frequency bands. He found that right parietal alpha mu oscillations were significantly more coupled in periods of spontaneous synchronization. Both results point towards the relevance of these patterns for the mirror neuron system. A similar paradigm - also investigating alpha-band activity - was used by Naeem et al. (2012a b). However, they did not replicate Tognoli's approach but focused on broader frequency bands in the mu range in different coordination contexts (intrinsic, in-phase, antiphase), suggesting functional discrimination of lower $(8-10 \mathrm{~Hz})$ and upper $(10-12 \mathrm{~Hz})$ $\mathrm{mu}$ band (Naeem et al. 2012a). While the former seems to reflect general attentional processes, the latter is modulated by task and hemisphere: in the left hemisphere, the top mu band is present 
during imitation, while in the right hemisphere, it is involved in perceptual-motor discrimination. Based on this, the authors suggest a right hemispheric circuit that modulates the way the actions of others are processed concerning the desired coordination mode (Naeem et al. 2012b). In another study that focused on the directionality of interaction, the subject associated as the leader showed a characteristic suppression of frontal alpha activity, possibly representing enhanced cognitive control and planning (Konvalinka et al., 2014). Manipulating the neural synchronization between the participants with tACS, two studies directly explored the impact of phase-coupled neural oscillations on behavioral performance. Results indicate that in-phase beta but not alpha or theta stimulation across the respective motor cortices facilitated tapping entrainment (Novembre et al. 2017, Szymanski et al. 2017a). However, it was not yet shown whether this effect could be replicated in EEG studies.

In contrast to such minimalistic experimental set-ups, several studies applied hyperscanning in cognitively more demanding and also more naturalistic settings. Recording two subjects interacting in a finger tapping imitation task, Holper et al. (2012) observed increased functional connectivity between two interacting brains. Social aspects modulated even unconscious fingertip movement synchronization: Yun et al. (2012) found that after having cooperated in an induced imitation task, the patterns of unconscious finger movement across two subjects became more synchronized. On a neural level, this change went along with increased theta and beta band phase locking across different regions, including the inferior frontal gyrus (IFG), anterior cingulate cortex (ACC), and ventromedial prefrontal cortex (vmPFC). In this context, the researchers associated the observed activity patterns with implicit social processing.

In a cognitively more demanding task, Lindenberger et al. (2009) investigated pairs of guitarists playing a melody together. Similarly, they reported that coordinated actions between the subjects involved oscillatory couplings between the two brains. During coordinated play onset, they found significantly increased phase synchronization between the two brains, primarily over frontal-central connections. The synchronization was exceptionally robust in low-frequency ranges, between 0.5 and $7.5 \mathrm{~Hz}$, with a maximum in the theta frequency at $3.3 \mathrm{~Hz}$. This result contradicts previously mentioned studies that primarily report dominant alpha synchronization during interpersonal action coordination. The authors, however, note that the observed couplings might merely reflect similarities in the temporal structure of the individual's perception and action. Accordingly, it is still not clear whether the neural coupling causes the effective movement coordination between the pairs. Rather than reporting specific brain areas and frequency bands, the studies mentioned above suggest that inter-brain connectivity through interpersonally coupled brain oscillations facilitates complex interpersonal action coordination.

Social aspects of action coordination, i.e., the influence of social connectedness and social character traits on synchronization performance, is another topic where multiple brain recordings provide new valuable insights. On a hormonal level, $\mathrm{Mu}$ et al. (2016) could show that oxytocin, intranasally administered, significantly facilitates neural synchronization in the alpha band and thus effectively supports movement coordination. Addressing the influence of the social connectedness between pairs, Pan et al. (2017) compared the performance of lovers to strangers and friends in a 
simple coordination task. Between the lover's brains, they indeed found a significantly increased synchronization. More specifically, they report that the right frontoparietal network is involved in romantic processing and social cognition. At the same time, lovers also showed a significant increase in coordination performance. Since oxytocin is strongly associated with social bondings, especially in romantic relations, this result supports $\mathrm{Mu}$ et al. (2016), indicating the human hormone's facilitating effect on interpersonal action coordination. Applying the same simple interaction task, $\mathrm{Hu}$ et al. (2017) found a correlation between the prosocial inclination of the subjects and their respective inter-brain synchronization. All in all, these studies support the evidence that social traits and the ability to synchronize in interpersonal coordination are strongly connected.

The current hyperscanning research on synchronization and coordination reports neural (synchronization) effects in various areas and frequency bands, although exploring very similar tasks. There are two groups of findings: The first group reports inter-subject neural couplings in frontal and parietal regions that are associated with better action synchronization (theta, alpha, and beta frequency). The second group of findings focuses on mechanisms that are not coupled across individuals but correspond to how a single brain processes incoming stimuli in a coordination context (Konvalinka et al., 2014, Naeem et al., 2012a b; Tognoli et al., 2007). Interestingly, these within-brain effects were also reported at frontal and centro-parietal sides in the alpha range. They all indicate that interpersonal action synchronization is accompanied by neuronal coupling of primarily frontal and centro-parietal areas in lower frequencies. However, concerning prominent frequency bands related with movement synchronization, the reported results do not seem to be conclusive: while some effects were mainly within the alpha $(10-12 \mathrm{~Hz})$ and beta ( $20 \mathrm{~Hz})$ range, others specifically exclude the alpha range and instead emphasize a synchronization in the theta frequency (Lindenberger et al., 2009; Novembre et al., 2017; Yun et al., 2012).

Apart from this, prosocial character traits such as the social connectedness of the dyads influence the effectiveness of synchronization. However, since many of the mentioned studies had less than ten pairs of subjects, more work is needed to ensure and replicate the results.

\section{Music}

Musical performances offer attractive experimental conditions since such performances combine intrapersonal action coordination and interpersonal action synchronization as well as continuous interaction. The advantages of musical settings for hyperscanning experiments are reviewed by Acquadro et al. (2016). A variety of experimental paradigms allow the investigation of different aspects of the interaction.

To investigate that inter-brain synchronization during an interaction is not only present due to the perception of the same ecological situation, researchers assign roles to the participants to investigate if complementary roles induce asymmetric patterns of brain synchronization. Sänger et al. (2012) investigate interpersonal action coordination using EEG hyperscanning of musical leader-follower duets playing a two-voiced piece of music repeatedly. They report within brain phase-locking modulated by the assigned role as well as extended within- and between-brain phase 
coherence during phases of high musical coordination. Because of the complementary voices of the piece, the phase coherence occurs in a situation where action and perception of the partners are not equal. Further, graph theory analyses show the presence of hyperbrain network structures. Later analysis of the same data by Sänger et al. (2013) allows investigation of the directionality of functional connectivity between the two brains. Results show directionality as a function of the musical roles. Pan et al. (2018) record brain activity of learner-instructor dyads during the acquisition of two songs using one of two learning methods (part learning vs. whole learning). The study records fNIRS data of bilateral fronto-temporoparietal regions. Across the part learning group, they report interpersonal brain synchronization during the learning periods, which was even able to predict the learning performance.

Furthermore, Granger causality analyses show coupling directionality from instructor to learner during a particular learning phase (teaching phase). The absence of interpersonal brain synchronization correlations in the whole learning group speaks against it as a mechanism of pure shared perception since both learning groups received equal sensory input and performed comparable actions. Synchronous oscillations are a present mechanism in leader-follower musical joint action tasks, the asynchronous nature of these signals gives rise to inter-brain synchrony partly as a mechanism of interactive task performance.

Other experimental designs investigate music without assigned roles, as this is the case in many musical contexts. While some experiments use existing music pieces, others engage in freer musical interactions like non-notated parts of songs or even improvisation. Novembre et al. (2016) use the structured properties of sheet music to manipulate familiarity and behavioral interpersonal synchronization during joint piano playing. With dyads of amateur piano players performing passages of two voiced joint playing with congruent and incongruent instructions for a later tempo change as well as alternating knowledge about the complementary voice, they report significant correlations between alpha suppression and congruent vs incongruent tempo instructions, in the case of the pianist being familiar with both voices of the passage. The authors conclude with the idea of alpha oscillations as neural processes regulating the balance between self-other integration and segregation, modulated by the compatibility of internal knowledge and external environmental information during joint action. After verifying EEG as a suitable method for hyperscanning in a musical context, Babiloni et al. (2011) used a hyperscanning paradigm to investigate empathy inside ensembles of musicians, playing a piece together (Babiloni et al. 2012). Alpha desynchronization in the right Brodmann area 44/45 during a video observation of their performance is positively correlated to the results of the Empathy Quotient Test score. Müller et al. (2013) investigate musical improvisation in dyads of guitarists. They analyze intra- and inter-brain synchrony during either a phase of joint improvisation or phases where one guitarist improvises while the other listens. They report high-frequency intra-brain connections as well as lower frequency inter-brain connections. Guitarists playing alone show stronger out-strength than the listening guitarist in the beta range; this difference was not present during joint improvisation. Osaka et al. (2014) compare fNIRS inter-brain coherence of participants during cooperative humming of a song with or without eye contact and single humming. Results indicate enhanced wavelet transform coherence inside the right 
inferior frontal cortex (IFC) during the non-face-to-face condition. A further study, Osaka et al. (2015), compares the inter-brain synchrony between dyads humming or singing a song, both again with or without visual contact, solo and joint. The left IFC shows increased synchronization for joint singing or humming, irrespective of the visual condition, while the right IFC shows increased synchronization specific to joint humming. The absence of synchrony in solo performances and aligned pseudo-pairs suggests the involvement of bilateral IFC in (musical) cooperation tasks. Inter-brain synchrony is a present mechanism even in more unstructured musical interactions, acting as a marker of interpersonal action coordination. Furthermore, experimental musical setups can be used for methodological analyses, as in the case of Zamm et al. (2018).

Altogether, the present results confirm musical paradigms as highly coordinative situations generating the ability to observe inter-brain synchrony as a mechanism of interpersonal action coordination, with a high potential for future research.

\section{Emotion and Affect}

Emotional regulation and affect play a crucial role in various forms of social interactions, for instance, the willingness to undertake joint actions with peers (Lopes et al., 2005) or in different types of prosocial behavior (Twenge et al., 2007). Neuroscientific studies measuring emotion and affect-based only on one participant's data lack the inter-brain connections among areas that might be involved in social behavior. In order to fill this gap, hyperscanning allows for recording inter-brain activity on emotions' onset and the simultaneous responses of interacting people.

To address the emotional component in social exchanges, several hyperscanning paradigms have been applied. Among these, setups have involved facial communication of affect Anders et al. 2011), mother-child interactions (Hirata et al., 2014; Levy et al., 2017), and goal-seeking tasks involving cooperation and competition conditions (Pan et al., 2017). Nonetheless, due to the complexity of the setups (i.e., Hirata et al. (2014)), hyperscanning studies have scarcely focused on the role of emotional regulation during joint actions (Ciaramidaro et al. 2018), leading in most of the cases to merely exploratory designs and vague hypotheses (Balconi and Vanutelli, 2017).

As an example of emotion processing during goal-oriented tasks, Hu et al. (2017) studied the prosocial behavior of dyads while performing a task in which participants performed coordinated and independent tasks across several trials. The authors found synchronized inter-brain activity only under the coordination task in the left middle frontal cortex (LMFC). This area has been commonly associated with memory, response inhibition, and people recognition during social interactions. Besides, Ciaramidaro et al. (2018) performed a study in which participants had the opportunity to distribute a quantity with a partner. A third participant (the observer) would judge the fairness of the distribution and decide whether to punish or not the participant who acted out the distribution. The dyads of participants involved in the exchange were composed of either human-human or computer-human. EEG data revealed higher inter-brain coherence of theta, alpha, and beta bands in the human-human condition between the observer and the receiver when the latter's action was rated as "hyper-unfair." Additionally, synchronous activity was also robust for PC-human fair 
interactions where the human participant received a fair reward. As the authors stated, situations with high emotional impact showed higher inter-brain synchronization.

In another experiment, Anders et al. (2011) investigated the emotional communication in romantic partners by observing the flow of information in male participants for emotional states perform by their female counterparts. They suggested that the neural activity of the perceiving partner can be successfully predicted from the neural activity of the sender's brain. This shared activation could only be found in dyads comprised of romantic partners but not in dyads of the sender and another participant different from her romantic couple. This finding suggests the development of reinforced neural paths present among sexual partners with highly emotional bindings.

Finally, some studies on emotions in social interaction have addressed the simultaneous measurement of inter-brain activity between mother and child. Levy et al. (2017), for instance, used a hyperscanning MEG set-up to measure the brain-to-brain activity of mother-child dyads by exposing them to video recordings of themselves performing positive and conflictual interactions. They found gamma activity in the superior temporal sulcus (STS) in interactions with behavioral synchrony (i.e., positive interactions). STS has been amply linked with social cognition, the theory of mind, and mirroring behavior. In the same line, Hirata et al. (2014) developed a hyperscanning MEG device that enables the mother and child to see each other's facial expressions during brain activity measurement. Although mainly of an explorative kind, these studies comprise a relevant background as pioneers on experimental designs to account for emotional interaction in hyperscanning setups.

Although not extensive, these studies highlight the moderator effect that the emotional component has in inter-brain activity in two scenarios. First, the closer the relationships between participants, the higher the inter-brain synchrony as observed in romantic couples and mother-child interactions. Second, inter-brain synchrony is higher for scenarios involving empathetic behavior, especially when these include an active emotional component. To sum up, the intensity of the emotional component modulates the synchronous neuronal activity during social interactions. Still, further research needs to be driven on this topic. For instance, the effect of well-studied emotions as stress or disgust has to be investigated. This can shed light on whether the impact of negative emotional interactions carries out more synchronize behavior than in the presence of emotions of a positive valence or if, instead, the modulatory effect of these might slightly depend on the sort of task.

\section{Cooperation and Competition}

Hyperscanning studies have addressed cooperative and competitive contexts under several methodological paradigms. These allow the study of both conditions within the same set-up. Therefore, participants can either cooperate or compete to achieve their goal meanwhile intra- and inter-brain activity is recorded. These include, for instance, the Prisoner's dilemma task (Babiloni et al. 2007a; De Vico Fallani et al., 2010), chicken's game (Astolfi et al., 2010), time estimation (Cui et al. 2012), turn-based interaction disk games (Liu et al., 2015, 2016b), Jenga (Liu et al., 2016a) or 
pong-game (Sinha et al. 2016).

Concerning intra-brain activity, hyperscanning studies reveal some commonalities of activation around the prefrontal cortex (PFC). For instance, during a Prisoner's Dilemma task, Babiloni et al. (2007a) found that $\mathrm{mPFC}$ is active during all the conditions (i.e., cooperation, defect, and tit-for-tat). In contrast, ACC is only activated when participants defect. In general, the global integration of brain areas was higher under the competitive condition than in cooperation and tit-for-tat. This is in line with findings by Astolfi et al. (2010), in which defect and tit-for-tat conditions obtained higher activity than for the cooperative condition in beta-band EEG recordings. $\mathrm{mPFC}$ has been generally related to social interaction supporting the constant activation observed during all conditions. On the other side, ACC has been linked to the theory of mind, indicating that an extra effort is needed to predict the opponent's behavior under competitive interactions. In another scenario, Liu et al. (2015, 2016b) performed a turn-based interaction in a computerized 2-people game. Participants took turns to be either a builder or a helper/obstructer partner, while brain activity was recorded using fNIRS. They found significant activation in IFG in builders during the cooperation condition but not when their partners were competing. A similar set-up was used by Liu et al. (2016a) in which a significantly higher activity was found in the obstructors' rIFG area. However, in both studies, no effect was found for helpers; that is, no "cooperated effect" was revealed. rIFG has been linked to empathy and intention understanding during interpersonal interactions. In this sense, results show a need for higher empathy when guidance is necessary to achieve a common goal. On the other side, when it comes to hinder other's performance, the understanding of an opponent's intentions plays a crucial role.

On an inter-brain level, the activation in PFC seems to be modulated by the condition and nature of the task. For instance, in the before-mentioned set-up, Liu et al. (2015, 2016b) found a significant inter-brain synchrony only in builder-obstructor pairs. Additionally, Liu et al. (2016a) observed active inter-brain synchrony in the posterior region of the right middle and superior frontal gyrus, particularly Brodmann area 8 (BA8), during cooperative and obstructive interaction (but not in the parallel game and talking condition). Inter-brain synchrony was also observed only during cooperative interaction in the dorsomedial prefrontal cortex (dmPFC), in particular, Brodmann area 9 (BA9). Since participants are performing a joint activity, motor execution has to be synchronized. This is in line with previous findings linking PFC with functions as planning and motor execution. On the contrary, other studies reported stronger synchronized inter-brain activity in cooperative contexts. As shown by De Vico Fallani et al. (2010) and Babiloni et al. (2007a) performing Prisoner's dilemma set-ups, hyper brain networks in competitive brains have fewer links and have overall higher modularity than in tit-for-tat and cooperative couples. Furthermore, Cui et al. (2012) found increased coherence between signals measured over the right superior frontal cortices between two brains in cooperative and not during competitive behavior. Supporting these findings, Sinha et al. (2016) reported significantly higher inter-brain synchrony between the subjects when they cooperated as compared to the competitive scenario. Additionally, we found that inter-brain synchrony was enhanced considerably when the subjects were physically separated, i.e., they cooperated via an intranet network. This is in contrast with Liu's (Liu et al., 2016a, 2015, 2016b) findings of 
synchronized activity in dmPFC in competitive contexts. This might be because different set-ups require synchronized activation under different conditions. For instance, a task like the prisoner dilemma needs a higher understanding of other's intentions when participants decide to cooperate.

All in all, hyperscanning studies confirm previous findings on the crucial role of dmPFC in collective behavior. However, the strength of this synchronized activation in dmPFC depends not only on the condition (i.e., cooperation and competition) but also on the specific kind of task as well. For instance, tasks like turn-taking games (e.g., Jenga) that require the prediction of the opponent's actions, demand a higher level of the theory of mind processing. On the other hand, tasks like the prisoner's dilemma imply empathy/theory of mind during the cooperative scenarios, and these differences are also reflected in between brain analysis.

\section{Games and decision making}

Overall studies in the field of games and decision making have shown that their neural underpinnings involve a network of regions. They are ranging from the medial frontal cortex (MFC), superior temporal sulcus (STS), to the temporoparietal junction (TPJ). Throughout the last years of research in the field of interactive decision making in games, a specialization of focus took place, as the first studies focused on areas being active in simple games, like the game theory. However, the first investigations to test the neural basis of social interaction used the game theory, as it allows us to define a social situation in which one may lose or profit. Babiloni et al. (2007a) demonstrate that a cooperative social interaction activates the reward circuitry. Noncooperative behavior, in contrast, does not. Their findings suggest a strong activation of the ACC and the cingulate motor area (CMA). The results point out the importance of the ACC, especially for leaders. In their case, the person who plays the first card on the deck.

Besides, Babiloni et al. (2006) presented EEG hyperscanning as a new and valid methodology to address brain activity of a group during real-life social interaction, the 'spirit of the group'. Building upon the findings from Babiloni et al. (2006). addressed social interaction during a game. The aim this time was to measure the neural activity of different brains simultaneously — particularly neural processes generated by social cooperation or competition. The results are similar, also providing evidence for the ACC and the CMA to be maximally active (Babiloni et al., 2007b). One other early experiment in the field of decision making was performed by Tomlin $(2006)$. They investigated the impact of personal and impersonal situations by using fMRI hyperscanning. Their findings are in line with the results by Babiloni et al. (2007a), as the dorsal anterior cingulate cortex responded strongly to their set up. Furthermore, cingulate and paracingulate cortices appear to contribute to social cognition and decision-making.

Further, Tomlin (2006) add the possibility that other variables in the social domain may impact outcomes in this area, like the belief in 'me' or 'not me'. Also, Yun et al. (2008) studied social decision making by using the Ultimatum Game, as the experimental model offers the estimation of, e.g., fairness or mind-reading, which has been used before as well (Sanfey, 2003) - also mentioning, as other authors do as well, the umbrella term 'theory of mind', showing how wide the topic can be 
interpreted. Their results suggest high-frequency oscillations in frontocentral regions, indicating that social interaction is closely related to this area. Investigating the effect of gender in cooperative and noncooperative situations, Cheng et al. (2015) used fNIRS and revealed that task-related coherence in brain activity. This was evident in regions of the frontal cortex, especially when opposite-sex partners are cooperating. The last study to mention here is the one by Zhang et al. (2017), as they provide an overview of research from the last years and taking another variable in their focus deception. In their study, they used fNIRS hyperscanning to measure pairs of participants in a two-person gambling card-game simultaneously. Their findings provide higher TPJ activation in deceptive acts, compared to honest ones. Further, they assume that STS may play a critical role in spontaneous deception. Decision making in games offers a well-controlled environment to investigate decision making. Future research has to uncover the precise influence of known and not know partner, and the differentiation between cooperation and competition. Furthermore, influences like facial expression or gestures, are worth considering.

\section{Action representation and Joint attention}

Whenever we socially interact with others, we have to coordinate our actions with those of our partners precisely. For successful joint action, we need to understand our partner's intentions and combine it with our action plan - always anticipating, attending, and adapting. In this context, joint attention provides the basis for shared awareness of common objects and goals, that is required to join our actions with others effectively. When studying neural mechanisms underlying these cognitive abilities, hyperscanning research provides new opportunities to investigate the intra- and inter-brain effects that accompany joint action. Setups reach from pure natural eye-to-eye contact and mutual visual search to more demanding joint musical performance.

Considering mutual gaze as the communicative context in which joint attention is initiated, Hirsch et al. (2017) investigated the neural effects of natural eye-to-eye contact via fNIRS. Comparing 'online' interactive eye-to-eye-contact with an 'offline' non-interactive eye-to-picture condition, they report a broad neural network reacting sensitive to interactive mutual gaze: During online eye-to-eye contact the hemodynamic signals of left frontal (pre- and supplementary Motor Cortex), and temporal-parietal regions displayed a higher functional connectivity within brains as well as increased synchronization between brains. This network vastly overlaps with regions associated with language perception and interpretation (i.e., Broca's and Wernicke's regions). Due the this, Hirsch and his team suppose that natural eye-to-eye-contact actively incorporates face-to-language processing. The cross-brain coherence observed in these areas supports this claim, indicating that the rapid online exchange of information between the brains enabling language processing is also communicatively active during mutual gaze.

Further research investigating mutual gaze, use similar experimental paradigms: They observed the brain activity of two subjects, interacting in a non-verbal joint attention task (Koike et al., 2016 Lachat et al., 2012, Saito et al., 2010). Here, subjects had to mutually attend target objects either by following the partner's gaze, by self-initiating the common gaze direction or by following an 
external cue. In the hyperscanning fMRI study of Saito et al. (2010), during moments of shared attention, paired subjects showed significantly higher inter-brain correlations in the IFG. They, therefore, concluded that observed inter-brain synchronization in the right IFG facilitates the formation of shared representations, enabling the incorporation of shared intentions by internalizing the other's intentions.

These findings closely relate to the reports of an extended fMRI study by Koike et al. (2016). In this experiment, the researches additionally examined the eye-blink synchronization between the subjects, considering them as an index of joint attention. Alternating between mutual gaze and joint attention tasks, dyads displayed increased synchronization of eye-blinks and right IFG activity when they have been previously engaged in a joint attention task. The researchers take this as an indication that the inter-personal neural synchronization through joint attention can be learned and, therefore, be maintained in the social memory. Similar to Saito et al. (2010), the study also reported significant inter-brain synchronization in the right IFG in the context of initiating as well as responding to joint attention. This synchronized activity also correlated positively with enhanced eye-blink synchronization. Importantly, in a video control condition, where participants did not see their partners as a live-recording, the right IFG showed no activity. From these results, the study infers that the right IFG acts as an interface between the self and the other: It is thus thought to coordinate constant shifts between central-executive and default-mode networks, moving attention between oneself and the partner. This fits well with Saito et al. (2010), which associates the synchronized activity of the right IFG with the formation of shared representations between subjects.

Applying dual EEG to compare the neural activity of socially driven versus color driven gaze direction, Lachat et al. (2012) based their research on different brain oscillations. They focused on frequency bands around $10 \mathrm{~Hz}$ over parieto-occipital and centro-parietal since this activity is generally associated with social coordination abilities. As previously expected, they found an attenuation of left-hemispheric alpha and mu rhythms by joint attention. This modulatory effect, however, was characteristic for mutually directed gaze in general, independent of the type of instruction, i.e., whether it was socially or color driven. The researchers interpret this suppression of the alpha mu rhythm as an indication for an 'attention mirroring system,' that allows subjects to orient their attention jointly. The left lateralization of this alpha mu attenuation contradicts previous research, where neural effects of social interaction are predominantly reported in the right hemisphere (Dumas et al., 2012, Koike et al., 2016, Novembre et al., 2016; Saito et al., 2010).

In contrast to these mutual gaze experiments, Szymanski et al. (2017b) compared individual performance with a joint performance during a visual search task. Here, the interaction between subjects was much more natural since verbal, gestural, and tactile communication could be used freely. The researchers tried to relate within and between brain neural dynamics to their respective team performance. Indeed, their results indicated that the overall team performance increased with intra- and inter-brain phase synchronization, especially in lower frequencies at frontal sites. Thus, local as well as between-brain phase synchronization is considered as a supportive factor for joint attention performance.

Beyond joint attention, the question of how two persons coordinate their actions with one 
another is subject of hyperscanning paradigms. Following the notion of co-representation (Sebanz et al. 2003), humans form an internal representation of another person's actions through common coding and mirror neuron mechanisms. This representation helps to adjust their actions in favor of a (joint) goal. However, the nature of human interactions is divers; relationships can be symmetric or complementary and emerge spontaneously or be predefined by the type of social situation. The question of how the representation of the self's and other's actions are modulated in these different contexts is the main subject of the studies discussed in the following.

Ménoret et al. (2014) investigated changes in electrophysiological patterns when we do not only observe an action but also co-act with our partner by performing a complementary task. They found that co-acting led to stronger movement-related beta suppression and more negative movement-related potentials at frontal sides in observers. This implies that co-acting goes along with a more intense representation of the other's action compared to mere observation. Sebanz et al. (2006) led two people to perform a go/nogo task alone or as a pair, sitting side by side. Each subject reacted to a different color cue, while a task-irrelevant stimulus pointed to a side either compatible or not compatible with the side on which the participant who was in turn to press the button was seated. Longer reaction times in the incompatible condition and a stronger Nogo P3 component at frontal and central electrodes in the group condition can be interpreted as a consequence of co-representing the partner's actions and the need to suppress own action-tendencies. Both Ménoret's and Sebanz's findings are in line with the concept of co-representation, indicating that observed as well as expected actions activate the according movement-related mechanisms within partners.

The relation between the anticipation of a partner's actions and dynamical entrainment was subject of Novembre et al. (2016). In his paradigm, subjects either familiar or unfamiliar with the partner's notes played a short melody together while tempo instructions were manipulated. Results showed that subjects unfamiliar with their partner's part acted more adaptive. On a neural level, modulations of alpha power at right centro-posterior sides were found: When subjects knew their partner's part, an incongruent tempo between the pianists led to a power increase, while good entrainment (based on congruent tempo instructions) led to an alpha power decrease. This allows the interpretation that alpha power modulates processes of self-other-integration and segregation. While the former is present when the tempo instructions match, the latter is observed when the tempo of the partner must be ignored in order to follow the instructions.

Dumas et al. (2012) aimed at distinguishing correlates of self-other-agency in a hand gesture imitation paradigm. Contrasting analyses across a broad frequency range $(0-48 \mathrm{~Hz})$ were used to extract differences between the conditions 'not moving and not observing', 'observing gestures passively, performing gestures alone, 'induced imitation,' and 'spontaneous imitation.' In induced imitation, the roles of model and follower were predefined by the experimenter, whereas they were established by the subjects in the spontaneous imitation condition. In the conditions where subjects performed and observed and performed gestures, a decrease of alpha mu power was observed over sensorimotor areas, including the temporal-parietal junction (TPJ). Hence alpha mu desynchronization might be a marker of action-perception-couplings. When subjects were primarily observing the action, passively, or as imitators, theta power increased. In the spontaneous condition, 
gamma was boosted across parietal regions, possibly representing the shared agency. The activation in parietal areas can be seen as a hint endorsing the relevance of TPJ for the agency and social interaction. Dumas et al. (2010) found an increased between brain phase locking in the alpha mu range during spontaneous synchronization.

While Dumas investigated random gestures, Schippers et al. (2010) addressed meaningful gestures used in a charade game. Gesturers, guessers, and control subjects that observed the gestures without guessing took turns in an fMRI scanner. Intending to find correlates of the mirror neuron system and mentalizing system, the researchers calculated the Granger causality between brains. The results support the relevance of the mirror neuron system for action representation, as the activity in the parietal region (associated with the mirror neuron system) of the gesturer predicted activity in the mirror neuron system and vmPFC (mentalizing system) of the observer. However, the involvement of the vmPFC was both statistically and theoretically less well funded than the mirror neuron system.

Based on these hyperscanning findings on joint attention and action representation, the relevance of the mirror neuron system and between brain connectivity in joint action representation gained further interest. This was shown directly by inter-brain-coherence (Dumas et al., 2010, Schippers et al. 2010) as well as indirectly utilizing observation-related-potentials and oscillatory patterns elicited during joint action contexts (Ménoret et al., 2014, Sebanz et al., 2006). There were also power modulations related to different modes of (joint) action found across a vast range of frequencies and regions, with alpha mu being the most prominent one, perhaps representing action-perception couplings (Dumas et al., 2012, Lachat et al., 2012, Novembre et al., 2016). When it comes to fluently segregating and integrating self- and other-related information during interpersonal coordination of actions, inter-brain synchronization seems to play a pivotal role.

To further validate the proposed hypotheses ascribed to these effects, repeating experiments in combination with different neuroimaging techniques might be useful to overcome the limitations each method has. This would also increase comparability across setups and thus allow for a complete picture and a better interpretation of the findings.

\section{Over two heads}

Naturalistic settings are attractive conditions for studying human interaction because, in such settings, interaction occurs without the intervention of the researcher, increasing the ecological validity of the findings. In the last years, researchers began to extend hyperscanning research towards multi-subject setups to increase the natural component of social interactions. Early group studies were EEG hyperscanning of four participants playing the Italian card game "Tressette" Astolfi et al. 2010, Babiloni et al. 2007b, 2006).

There are a variety of reasons for conducting experiments with a multi-subject design with different ideas of making the studied interaction more natural. Social behavior only evolves in the presence of other people, often groups. The presence of other people might enhance individual task performances (Wahn et al. 2018, 2019). In a dyadic setup, interactions might quickly become predictable. Extending the dyadic setup to larger groups may increase the complexity of the 
interaction due to the actions influencing more individuals generating more possible outcomes. Competition becomes more competitive, and cooperation tasks might become more complex, requiring better interaction of all members of the group. In the context of musical group performances, the structure of a leader and a follower often does no longer exist, playing in an ensemble requires continuous interaction of all members (Babiloni et al., 2012, 2011). In general, the roles of the participants in the interaction become less discrete. This is similar to many social interactions in daily life. Researchers use these properties for two kinds of experimental design. Some apply findings, conducted from earlier experiments using dyadic design, to a group design to investigate whether these findings still hold under the more natural conditions. Other publications claim that the effects they want to observe can only be present inside a group interaction. Hyperscanning, hence, allows investigation of effects that are only present inside large groups like, for example, classrooms, allowing different and new research questions. Such experiments, therefore, observe social behavior inside a social setting. There thus exist two main categories of current multi-subject hyperscanning research.

Multi-subject hyperscanning experiments can be used to confirm results derived from less complex social situations in a more natural setting. Gevins et al. (2012) generate a measure to distinguish subjects under the influence of alcohol from others by their EEG data. The measure was derived from EEG data, recorded from non-interactive task performance. This measure is then applied to EEG data, simultaneously recorded from each participant of a cocktail party, and still correctly discriminates subjects under the influence of alcohol or placebos. Multi-subject Hyperscanning experiments hence offer a potential method for investigations regarding social behavior.

For other researchers, hyperscanning offers a new opportunity to precisely record human group interaction to investigate social dynamics. Dikker et al. (2017) investigate brain synchrony from a class of twelve high school students over one semester during regular classroom activities. The results suggest that the individuals that are less engaged with the classroom setting show lower brain to brain synchrony with the rest of the group. Nozawa et al. (2016) investigate brain synchrony inside twelve groups of four members playing a word chain game under a cooperative condition, reporting frontopolar interpersonal neural synchronization by natural and unstructured verbal communication. Results like these suggest that multi-subject hyperscanning experiments can also be conducted to observe the effects of social interactions directly.

\section{Intervention methods}

Intervention methods are especially appealing because properties of the object of investigation are directly manipulated: The activity of specific neural populations in the brain is up or downregulated by physiological or pharmacological means. This facilitates to relate changes in behavior to distinct neural processes of social interaction. Mu et al. (2016) applied EEG hyperscanning and studied the effects of oxytocin in males on the performance in a reciprocal synchronization task. The task was to synchronize a button press (varying delay in the second range) with the interaction partner or a 
computer. In contrast, Novembre et al. (2017) applied transcranial alternating current stimulation targeting at motor cortices of participants of each dyad. The authors compared behavioral measures for differences between in-phase and out-of-phase stimulation across subjects in a joint tapping paradigm. Similarly, Szymanski et al. (2017a) targeted the effects of same-phase-same-frequency hyper-tACS on the performance of participants in a joint drumming experiment. All three studies used synchronicity of behavior as a behavioral measure. Significant effects of Oxytocin on the mean alpha-band inter-brain PLV of posterior and central electrodes of males were found only for the social condition. However, most electrodes showed significant differences in this condition. In contrast, if participants synchronized their behavior to a computer, the difference between the treatment group and control was absent (Mu et al., 2016). Results from named tACS-studies show deviating results. Novembre et al. (2017) found higher inter-personal tapping synchrony for in-phase stimulation only for stimulation at $20 \mathrm{~Hz}$. In contrast, Szymanski et al. (2017a) did not find meaningful effects of in-phase stimulation on behavior. Future research may profit from the increase from a better understanding of intervention methods and theoretical grounding of expected and observed effects.

\section{Conclusions}

Taken together, in this review, we first presented methods that are used to measure the brain activity of two or more participants simultaneously. We discussed their advantages and disadvantages for studying different aspects of social interaction. Further, we reviewed the analysis methods that are used to study between brain networks. We listed different types of analyses that can contribute to various aspects of our understanding of the social brain. In the final section, we presented results of hyperscanning studies performed in the last two decades that focused on diverse cognitive functions and their neural underpinnings. In sum, with the evidence presented in this review, we tried to give an informed overview of the field and point out future avenues of research to foster insights into the interacting mind/brain.

\section{Conflict of Interest Statement}

The authors declare that the research was conducted in the absence of any commercial or financial relationships that could be construed as a potential conflict of interest.

\section{Author Contributions}

$\mathrm{AC}$ and PK: organized and supervised reading club at the University of Osnabrück to summarize and discuss Hyperscanning literature. AC, SE, AL, DM, MG, SS, FS, ZZR, PK: summarized all papers discussed in the review, wrote the review. AC and PK: draft and revision of the manuscript. 


\section{References}

Abe, M. O., Koike, T., Okazaki, S., Sugawara, S. K., Takahashi, K., Watanabe, K., and Sadato, N. (2019). Neural correlates of online cooperation during joint force production. NeuroImage, 191:150-161.

Acquadro, M. A. S., Congedo, M., and De Riddeer, D. (2016). Music Performance As an Experimental Approach to Hyperscanning Studies. Frontiers in Human Neuroscience, 10.

Ahn, S., Cho, H., Kwon, M., Kim, K., Kwon, H., Kim, B. S., Chang, W. S., Chang, J. W., and Jun, S. C. (2018). Interbrain phase synchronization during turn-taking verbal interaction-a hyperscanning study using simultaneous EEG/MEG: Synchronization During Turn-Taking Verbal Interaction. Human Brain Mapping, 39(1):171-188.

Anders, S., Heinzle, J., Weiskopf, N., Ethofer, T., and Haynes, J.-D. (2011). Flow of affective information between communicating brains. NeuroImage, 54(1):439-446.

Astolfi, L., Cincotti, F., Mattia, D., De Vico Fallani, F., Salinari, S., Vecchiato, G., Toppi, J., Wilke, C., Doud, A., Yuan, H., He, B., and Babiloni, F. (2010). Imaging the social brain: multi-subjects EEG recordings during the chicken's game. In 2010 Annual International Conference of the IEEE Engineering in Medicine and Biology, pages 1734-1737, Buenos Aires. IEEE.

Astolfi, L., Toppi, J., Borghini, G., Vecchiato, G., He, E. J., Roy, A., Cincotti, F., Salinari, S., Mattia, D., He, B., and Babiloni, F. (2012). Cortical activity and functional hyperconnectivity by simultaneous EEG recordings from interacting couples of professional pilots. In 2012 Annual International Conference of the IEEE Engineering in Medicine and Biology Society, pages 4752-4755, San Diego, CA. IEEE.

Astolfi, L., Toppi, J., Borghini, G., Vecchiato, G., Isabella, R., De Vico Fallani, F., Cincotti, F., Salinari, S., Mattia, D., He, B., Caltagirone, C., and Babiloni, F. (2011). Study of the functional hyperconnectivity between couples of pilots during flight simulation: An EEG hyperscanning study. In 2011 Annual International Conference of the IEEE Engineering in Medicine and Biology Society, pages 2338-2341, Boston, MA. IEEE. 
Babiloni, C., Buffo, P., Vecchio, F., Marzano, N., Del Percio, C., Spada, D., Rossi, S., Bruni, I., Rossini, P. M., and Perani, D. (2012). Brains "in concert": Frontal oscillatory alpha rhythms and empathy in professional musicians. NeuroImage, 60(1):105-116.

Babiloni, C., Vecchio, F., Infarinato, F., Buffo, P., Marzano, N., Spada, D., Rossi, S., Bruni, I., Rossini, P. M., and Perani, D. (2011). Simultaneous recording of electroencephalographic data in musicians playing in ensemble. Cortex, 47(9):1082-1090.

Babiloni, F., Astolfi, L., Cincotti, F., Mattia, D., Tocci, A., Tarantino, A., Marciani, M., Salinari, S., Gao, S., Colosimo, A., and De Vico Fallani, F. (2007a). Cortical Activity and Connectivity of Human Brain during the Prisoner's Dilemma: an EEG Hyperscanning Study. In 2007 29th Annual International Conference of the IEEE Engineering in Medicine and Biology Society, pages 4953-4956, Lyon, France. IEEE.

Babiloni, F., Cincotti, F., Mattia, D., De Vico Fallani, F., Tocci, A., Bianchi, L., Salinari, S., Marciani, M., Colosimo, A., and Astolfi, L. (2007b). High Resolution EEG Hyperscanning During a Card Game. In 2007 29th Annual International Conference of the IEEE Engineering in Medicine and Biology Society, pages 4957-4960, Lyon, France. IEEE.

Babiloni, F., Cincotti, F., Mattia, D., Mattiocco, M., Fallani, F. D. V., Tocci, A., Bianchi, L., Marciani, M. G., and Astolfi, L. (2006). Hypermethods for eeg hyperscanning. In 2006 International Conference of the IEEE Engineering in Medicine and Biology Society, pages 3666-3669. IEEE.

Baess, P., Zhdanov, A., Mandel, A., Parkkonen, L., Hirvenkari, L., Mäkelä, J. P., Jousmäki, V., and Hari, R. (2012). MEG dual scanning: a procedure to study real-time auditory interaction between two persons. Frontiers in Human Neuroscience, 6.

Baillet, S. (2017). Magnetoencephalography for brain electrophysiology and imaging. Nature Neuroscience, 20(3):327-339.

Balconi, M. and Vanutelli, M. E. (2017). Cooperation and Competition with Hyperscanning Methods: Review and Future Application to Emotion Domain. Frontiers in Computational Neuroscience, 11.

Bevilacqua, D., Davidesco, I., Wan, L., Chaloner, K., Rowland, J., Ding, M., Poeppel, D., and Dikker, S. (2019). Brain-to-Brain Synchrony and Learning Outcomes Vary by Student-Teacher Dynamics: Evidence from a Real-world Classroom Electroencephalography Study. Journal of Cognitive Neuroscience, 31(3):401-411.

Bilek, E., Ruf, M., Schäfer, A., Akdeniz, C., Calhoun, V. D., Schmahl, C., Demanuele, C., Tost, H., Kirsch, P., and Meyer-Lindenberg, A. (2015). Information flow between interacting human brains: Identification, validation, and relationship to social expertise. Proceedings of the National Academy of Sciences, 112(16):5207-5212. 
Bilek, E., Stößel, G., Schäfer, A., Clement, L., Ruf, M., Robnik, L., Neukel, C., Tost, H., Kirsch, P., and Meyer-Lindenberg, A. (2017). State-Dependent Cross-Brain Information Flow in Borderline Personality Disorder. JAMA Psychiatry, 74(9):949.

Boto, E., Holmes, N., Leggett, J., Roberts, G., Shah, V., Meyer, S. S., Muñoz, L. D., Mullinger, K. J., Tierney, T. M., Bestmann, S., Barnes, G. R., Bowtell, R., and Brookes, M. J. (2018). Moving magnetoencephalography towards real-world applications with a wearable system. Nature, $555(7698): 657-661$.

Cheng, X., Li, X., and Hu, Y. (2015). Synchronous brain activity during cooperative exchange depends on gender of partner: A fNIRS-based hyperscanning study: Synchronous Brain Activities. Human Brain Mapping, 36(6):2039-2048.

Ciaramidaro, A., Toppi, J., Casper, C., Freitag, C. M., Siniatchkin, M., and Astolfi, L. (2018). Multiple-Brain Connectivity During Third Party Punishment: an EEG Hyperscanning Study. Scientific Reports, 8(1).

Cipresso, P., Giglioli, I. A. C., Raya, M. A., and Riva, G. (2018). The Past, Present, and Future of Virtual and Augmented Reality Research: A Network and Cluster Analysis of the Literature. Frontiers in Psychology, 9.

Cui, X., Bryant, D. M., and Reiss, A. L. (2012). NIRS-based hyperscanning reveals increased interpersonal coherence in superior frontal cortex during cooperation. NeuroImage, $59(3): 2430-2437$.

De Vico Fallani, F., Nicosia, V., Sinatra, R., Astolfi, L., Cincotti, F., Mattia, D., Wilke, C., Doud, A., Latora, V., He, B., and Babiloni, F. (2010). Defecting or Not Defecting: How to "Read" Human Behavior during Cooperative Games by EEG Measurements. PLoS ONE, 5(12):e14187.

Dikker, S., Wan, L., Davidesco, I., Kaggen, L., Oostrik, M., McClintock, J., Rowland, J., Michalareas, G., Van Bavel, J. J., Ding, M., and Poeppel, D. (2017). Brain-to-Brain Synchrony Tracks Real-World Dynamic Group Interactions in the Classroom. Current Biology, 27(9):1375-1380.

Dodel, S., Cohn, J., Mersmann, J., Luu, P., Forsythe, C., and Jirsa, V. (2011). Brain Signatures of Team Performance. In Schmorrow, D. D. and Fidopiastis, C. M., editors, Foundations of Augmented Cognition. Directing the Future of Adaptive Systems, volume 6780, pages 288-297. Springer Berlin Heidelberg, Berlin, Heidelberg.

Dommer, L., Jäger, N., Scholkmann, F., Wolf, M., and Holper, L. (2012). Between-brain coherence during joint n-back task performance: A two-person functional near-infrared spectroscopy study. Behavioural Brain Research, 234(2):212-222.

Dumas, G., Lachat, F., Martinerie, J., Nadel, J., and George, N. (2011). From social behaviour to brain synchronization: Review and perspectives in hyperscanning. IRBM, 32(1):48-53. 
Dumas, G., Martinerie, J., Soussignan, R., and Nadel, J. (2012). Does the brain know who is at the origin of what in an imitative interaction? Frontiers in Human Neuroscience, 6.

Dumas, G., Nadel, J., Soussignan, R., Martinerie, J., and Garnero, L. (2010). Inter-Brain Synchronization during Social Interaction. PLoS ONE, 5(8):e12166.

Ehinger, B. V., Fischer, P., Gert, A. L., Kaufhold, L., Weber, F., Pipa, G., and König, P. (2014). Kinesthetic and vestibular information modulate alpha activity during spatial navigation: a mobile EEG study. Frontiers in Human Neuroscience, 8.

Eisenberger, N. I. (2003). Does Rejection Hurt? An fMRI Study of Social Exclusion. Science, $302(5643): 290-292$.

Ferrari, M. and Quaresima, V. (2012). A brief review on the history of human functional near-infrared spectroscopy (fNIRS) development and fields of application. NeuroImage, $63(2): 921-935$.

Funane, T., Kiguchi, M., Atsumori, H., Sato, H., Kubota, K., and Koizumi, H. (2011). Synchronous activity of two people's prefrontal cortices during a cooperative task measured by simultaneous near-infrared spectroscopy. Journal of Biomedical Optics, 16(7):077011.

Gevins, A., Chan, C. S., and Sam-Vargas, L. (2012). Towards Measuring Brain Function on Groups of People in the Real World. PLoS ONE, 7(9):e44676.

Glover, G. H. (2011). Overview of Functional Magnetic Resonance Imaging. Neurosurgery Clinics of North America, 22(2):133-139.

Grinsted, A., Moore, J. C., and Jevrejeva, S. (2004). Application of the cross wavelet transform and wavelet coherence to geophysical time series. Nonlinear Processes in Geophysics, 11(5/6):561-566.

Hari, R., Himberg, T., Nummenmaa, L., Hämäläinen, M., and Parkkonen, L. (2013). Synchrony of brains and bodies during implicit interpersonal interaction. Trends in Cognitive Sciences, $17(3): 105-106$.

Hari, R. and Kujala, M. V. (2009). Brain Basis of Human Social Interaction: From Concepts to Brain Imaging. Physiological Reviews, 89(2):453-479.

Hirata, M., Ikeda, T., Kikuchi, M., Kimura, T., Hiraishi, H., Yoshimura, Y., and Asada, M. (2014). Hyperscanning MEG for understanding mother-child cerebral interactions. Frontiers in Human Neuroscience, 8.

Hirsch, J., Zhang, X., Noah, J. A., and Ono, Y. (2017). Frontal temporal and parietal systems synchronize within and across brains during live eye-to-eye contact. NeuroImage, 157:314-330.

Holper, L., Scholkmann, F., and Wolf, M. (2012). Between-brain connectivity during imitation measured by fNIRS. NeuroImage, 63(1):212-222. 
Hu, Y., Hu, Y., Li, X., Pan, Y., and Cheng, X. (2017). Brain-to-brain synchronization across two persons predicts mutual prosociality. Social Cognitive and Affective Neuroscience, 12(12):1835-1844.

Hu, Y., Pan, Y., Shi, X., Cai, Q., Li, X., and Cheng, X. (2018). Inter-brain synchrony and cooperation context in interactive decision making. Biological Psychology, 133:54-62.

Ikeda, S., Nozawa, T., Yokoyama, R., Miyazaki, A., Sasaki, Y., Sakaki, K., and Kawashima, R. (2017). Steady Beat Sound Facilitates both Coordinated Group Walking and Inter-Subject Neural Synchrony. Frontiers in Human Neuroscience, 11.

Jiang, J., Chen, C., Dai, B., Shi, G., Ding, G., Liu, L., and Lu, C. (2015). Leader emergence through interpersonal neural synchronization. Proceedings of the National Academy of Sciences, 112(14):4274-4279.

Jiang, J., Dai, B., Peng, D., Zhu, C., Liu, L., and Lu, C. (2012). Neural Synchronization during Face-to-Face Communication. Journal of Neuroscience, 32(45):16064-16069.

Kawasaki, M., Yamada, Y., Ushiku, Y., Miyauchi, E., and Yamaguchi, Y. (2013). Inter-brain synchronization during coordination of speech rhythm in human-to-human social interaction. Scientific Reports, 3(1).

King-Casas, B. (2005). Getting to Know You: Reputation and Trust in a Two-Person Economic Exchange. Science, 308(5718):78-83.

Kinreich, S., Djalovski, A., Kraus, L., Louzoun, Y., and Feldman, R. (2017). Brain-to-Brain Synchrony during Naturalistic Social Interactions. Scientific Reports, 7(1).

Koike, T., Sumiya, M., Nakagawa, E., Okazaki, S., and Sadato, N. (2019). What Makes Eye Contact Special? Neural Substrates of On-Line Mutual Eye-Gaze: A Hyperscanning fMRI Study. eneuro, 6(1):ENEURO.0284-18.2019.

Koike, T., Tanabe, H. C., Okazaki, S., Nakagawa, E., Sasaki, A. T., Shimada, K., Sugawara, S. K., Takahashi, H. K., Yoshihara, K., Bosch-Bayard, J., and Sadato, N. (2016). Neural substrates of shared attention as social memory: A hyperscanning functional magnetic resonance imaging study. NeuroImage, 125:401-412.

Koike, T., Tanabe, H. C., and Sadato, N. (2015). Hyperscanning neuroimaging technique to reveal the "two-in-one" system in social interactions. Neuroscience Research, 90:25-32.

Konvalinka, I., Bauer, M., Stahlhut, C., Hansen, L. K., Roepstorff, A., and Frith, C. D. (2014). Frontal alpha oscillations distinguish leaders from followers: Multivariate decoding of mutually interacting brains. NeuroImage, 94:79-88.

Lachat, F., Hugueville, L., Lemaréchal, J.-D., Conty, L., and George, N. (2012). Oscillatory Brain Correlates of Live Joint Attention: A Dual-EEG Study. Frontiers in Human Neuroscience, 6. 
Levy, J., Goldstein, A., and Feldman, R. (2017). Perception of social synchrony induces mother-child gamma coupling in the social brain. Social Cognitive and Affective Neuroscience, 12(7):1036-1046.

Lindenberger, U., Li, S.-C., Gruber, W., and Müller, V. (2009). Brains swinging in concert: cortical phase synchronization while playing guitar. BMC Neuroscience, 10(1).

Liu, N., Mok, C., Witt, E. E., Pradhan, A. H., Chen, J. E., and Reiss, A. L. (2016a). NIRS-Based Hyperscanning Reveals Inter-brain Neural Synchronization during Cooperative Jenga Game with Face-to-Face Communication. Frontiers in Human Neuroscience, 10.

Liu, T., Saito, H., and Oi, M. (2015). Role of the right inferior frontal gyrus in turn-based cooperation and competition: A near-infrared spectroscopy study. Brain and Cognition, 99:17-23.

Liu, T., Saito, H., and Oi, M. (2016b). Obstruction increases activation in the right inferior frontal gyrus. Social Neuroscience, 11(4):344-352.

Lopes, P. N., Salovey, P., Côté, S., and Beers, M. (2005). Emotion Regulation Abilities and the Quality of Social Interaction. Emotion, 5(1):113-118.

Luck, S. J. and Hillyard, S. A. (1994). Spatial filtering during visual search: evidence from human electrophysiology. Journal of Experimental Psychology: Human Perception and Performance, 20(5):1000.

Melnik, A., Legkov, P., Izdebski, K., Kärcher, S. M., Hairston, W. D., Ferris, D. P., and König, P. (2017). Systems, Subjects, Sessions: To What Extent Do These Factors Influence EEG Data? Frontiers in Human Neuroscience, 11.

Michel, C. M. and Brunet, D. (2019). EEG Source Imaging: A Practical Review of the Analysis Steps. Frontiers in Neurology, 10.

Montague, P. (2002). Hyperscanning: Simultaneous fMRI during Linked Social Interactions. NeuroImage, 16(4):1159-1164.

$\mathrm{Mu}$, Y., Guo, C., and Han, S. (2016). Oxytocin enhances inter-brain synchrony during social coordination in male adults. Social Cognitive and Affective Neuroscience, 11(12):1882-1893.

Ménoret, M., Varnet, L., Fargier, R., Cheylus, A., Curie, A., des Portes, V., Nazir, T. A., and Paulignan, Y. (2014). Neural correlates of non-verbal social interactions: A dual-EEG study. Neuropsychologia, 55:85-97.

Müller, V. and Lindenberger, U. (2014). Hyper-Brain Networks Support Romantic Kissing in Humans. PLoS ONE, 9(11):e112080.

Müller, V., Sänger, J., and Lindenberger, U. (2013). Intra- and Inter-Brain Synchronization during Musical Improvisation on the Guitar. PLoS ONE, 8(9):e73852. 
Naeem, M., Prasad, G., Watson, D. R., and Kelso, J. S. (2012a). Electrophysiological signatures of intentional social coordination in the 10-12hz range. NeuroImage, 59(2):1795-1803.

Naeem, M., Prasad, G., Watson, D. R., and Kelso, J. S. (2012b). Functional dissociation of brain rhythms in social coordination. Clinical Neurophysiology, 123(9):1789-1797.

Novembre, G., Knoblich, G., Dunne, L., and Keller, P. E. (2017). Interpersonal synchrony enhanced through $20 \mathrm{~Hz}$ phase-coupled dual brain stimulation. Social Cognitive and Affective Neuroscience, 12(4):662-670.

Novembre, G., Sammler, D., and Keller, P. E. (2016). Neural alpha oscillations index the balance between self-other integration and segregation in real-time joint action. Neuropsychologia, 89:414-425.

Nozawa, T., Sasaki, Y., Sakaki, K., Yokoyama, R., and Kawashima, R. (2016). Interpersonal frontopolar neural synchronization in group communication: An exploration toward fNIRS hyperscanning of natural interactions. NeuroImage, 133:484-497.

Oliveira, A. S., Schlink, B. R., Hairston, W. D., König, P., and Ferris, D. P. (2016). Induction and separation of motion artifacts in EEG data using a mobile phantom head device. Journal of Neural Engineering, 13(3):036014.

Osaka, N., Minamoto, T., Yaoi, K., Azuma, M., and Osaka, M. (2014). Neural Synchronization During Cooperated Humming: A Hyperscanning Study Using fNIRS. Procedia - Social and Behavioral Sciences, 126:241-243.

Osaka, N., Minamoto, T., Yaoi, K., Azuma, M., Shimada, Y. M., and Osaka, M. (2015). How Two Brains Make One Synchronized Mind in the Inferior Frontal Cortex: fNIRS-Based Hyperscanning During Cooperative Singing. Frontiers in Psychology, 6.

Pan, Y., Cheng, X., Zhang, Z., Li, X., and Hu, Y. (2017). Cooperation in lovers: An fNIRS-based hyperscanning study: Cooperation in Lovers. Human Brain Mapping, 38(2):831-841.

Pan, Y., Novembre, G., Song, B., Li, X., and Hu, Y. (2018). Interpersonal synchronization of inferior frontal cortices tracks social interactive learning of a song. NeuroImage, 183:280-290.

Pérez, A., Carreiras, M., and Duñabeitia, J. A. (2017). Brain-to-brain entrainment: EEG interbrain synchronization while speaking and listening. Scientific Reports, 7(1).

Quaresima, V. and Ferrari, M. (2019). Functional Near-Infrared Spectroscopy (fNIRS) for Assessing Cerebral Cortex Function During Human Behavior in Natural/Social Situations: A Concise Review. Organizational Research Methods, 22(1):46-68.

Reindl, V., Gerloff, C., Scharke, W., and Konrad, K. (2018). Brain-to-brain synchrony in parent-child dyads and the relationship with emotion regulation revealed by fNIRS-based hyperscanning. NeuroImage, 178:493-502. 
Saito, D. N., Tanabe, H. C., Izuma, K., Hayashi, M. J., Morito, Y., Komeda, H., Uchiyama, H., Kosaka, H., Okazawa, H., Fujibayashi, Y., and Sadato, N. (2010). "Stay Tuned": Inter-Individual Neural Synchronization During Mutual Gaze and Joint Attention. Frontiers in Integrative Neuroscience, 4.

Sanfey, A. G. (2003). The Neural Basis of Economic Decision-Making in the Ultimatum Game. Science, 300(5626):1755-1758.

Schilbach, L., Timmermans, B., Reddy, V., Costall, A., Bente, G., Schlicht, T., and Vogeley, K. (2013). Toward a second-person neuroscience. Behavioral and Brain Sciences, 36(4):393-414.

Schippers, M. B., Roebroeck, A., Renken, R., Nanetti, L., and Keysers, C. (2010). Mapping the information flow from one brain to another during gestural communication. Proceedings of the National Academy of Sciences, 107(20):9388-9393.

Scholkmann, F., Holper, L., Wolf, U., and Wolf, M. (2013). A new methodical approach in neuroscience: assessing inter-personal brain coupling using functional near-infrared imaging (fNIRI) hyperscanning. Frontiers in Human Neuroscience, 7.

Sebanz, N., Knoblich, G., and Prinz, W. (2003). Representing others' actions: just like one's own? Cognition, 88(3):B11-B21.

Sebanz, N., Knoblich, G., Prinz, W., and Wascher, E. (2006). Twin Peaks: An ERP Study of Action Planning and Control in Coacting Individuals. Journal of Cognitive Neuroscience, 18(5):859-870.

Sinha, N., Maszczyk, T., Zhang Wanxuan, Tan, J., and Dauwels, J. (2016). EEG hyperscanning study of inter-brain synchrony during cooperative and competitive interaction. In 2016 IEEE International Conference on Systems, Man, and Cybernetics (SMC), pages 004813-004818, Budapest, Hungary. IEEE.

Spiegelhalder, K., Ohlendorf, S., Regen, W., Feige, B., Tebartz van Elst, L., Weiller, C., Hennig, J., Berger, M., and Tüscher, O. (2014). Interindividual synchronization of brain activity during live verbal communication. Behavioural Brain Research, 258:75-79.

Szymanski, C., Müller, V., Brick, T. R., von Oertzen, T., and Lindenberger, U. (2017a).

Hyper-Transcranial Alternating Current Stimulation: Experimental Manipulation of Inter-Brain Synchrony. Frontiers in Human Neuroscience, 11.

Szymanski, C., Pesquita, A., Brennan, A. A., Perdikis, D., Enns, J. T., Brick, T. R., Müller, V., and Lindenberger, U. (2017b). Teams on the same wavelength perform better: Inter-brain phase synchronization constitutes a neural substrate for social facilitation. NeuroImage, 152:425-436.

Sänger, J., Müller, V., and Lindenberger, U. (2012). Intra- and interbrain synchronization and network properties when playing guitar in duets. Frontiers in Human Neuroscience, 6. 
Sänger, J., Müller, V., and Lindenberger, U. (2013). Directionality in hyperbrain networks discriminates between leaders and followers in guitar duets. Frontiers in Human Neuroscience, 7.

Tanabe, H. C., Kosaka, H., Saito, D. N., Koike, T., Hayashi, M. J., Izuma, K., Komeda, H., Ishitobi, M., Omori, M., Munesue, T., Okazawa, H., Wada, Y., and Sadato, N. (2012). Hard to "tune in": neural mechanisms of live face-to-face interaction with high-functioning autistic spectrum disorder. Frontiers in Human Neuroscience, 6.

Tang, H., Mai, X., Wang, S., Zhu, C., Krueger, F., and Liu, C. (2016). Interpersonal brain synchronization in the right temporo-parietal junction during face-to-face economic exchange. Social Cognitive and Affective Neuroscience, 11(1):23-32.

Tognoli, E., Lagarde, J., DeGuzman, G. C., and Kelso, J. A. S. (2007). The phi complex as a neuromarker of human social coordination. Proceedings of the National Academy of Sciences, 104(19):8190-8195.

Tomlin, D. (2006). Agent-Specific Responses in the Cingulate Cortex During Economic Exchanges. Science, 312(5776):1047-1050.

Tomlin, D., Nedic, A., Prentice, D. A., Holmes, P., and Cohen, J. D. (2013). The Neural Substrates of Social Influence on Decision Making. PLoS ONE, 8(1):e52630.

Twenge, J. M., Baumeister, R. F., DeWall, C. N., Ciarocco, N. J., and Bartels, J. M. (2007). Social exclusion decreases prosocial behavior. Journal of Personality and Social Psychology, 92(1):56-66.

Wahn, B., Czeszumski, A., and König, P. (2018). Performance similarities predict collective benefits in dyadic and triadic joint visual search. PloS one, 13(1):e0191179.

Wahn, B., Czeszumski, A., Labusch, M., Kingstone, A., and König, P. (2019). Dyadic and triadic search: Benefits, costs, and predictors of group performance. PsyArXiv.

Wang, C., Zhang, T., Shan, Z., Liu, J., Yuan, D., and Li, X. (2019). Dynamic interpersonal neural synchronization underlying pain-induced cooperation in females. Human Brain Mapping.

Wen, X., Mo, J., and Ding, M. (2012). Exploring resting-state functional connectivity with total interdependence. NeuroImage, 60(2):1587-1595.

Yun, K., Chung, D., and Jeong, J. (2008). Emotional Interactions in Human Decision Making using EEG Hyperscanning. In International Conference of Cognitive Science, page 4.

Yun, K., Watanabe, K., and Shimojo, S. (2012). Interpersonal body and neural synchronization as a marker of implicit social interaction. Scientific Reports, 2(1).

Zamm, A., Debener, S., Bauer, A.-K. R., Bleichner, M. G., Demos, A. P., and Palmer, C. (2018). Amplitude envelope correlations measure synchronous cortical oscillations in performing musicians: Amplitude envelopes measure inter-brain synchrony. Annals of the New York Academy of Sciences, 1423(1):251-263. 
Zhang, M., Liu, T., Pelowski, M., and Yu, D. (2017). Gender difference in spontaneous deception: A hyperscanning study using functional near-infrared spectroscopy. Scientific Reports, 7(1). 